\title{
MIMO System Reduction Using Modified Pole Clustering and Genetic Algorithm
}

\author{
C. B. Vishwakarma ${ }^{1}$ and R. Prasad ${ }^{2}$ \\ ${ }^{1}$ Faculty of Engineering and Technology, Gurukul Kangri University, Haridwar 249-404, India \\ ${ }^{2}$ Department of Electrical Engineering, Indian Institute of Technology, Roorkee 247-667, India
}

Correspondence should be addressed to C. B. Vishwakarma, cbv_gkv@yahoo.co.in

Received 14 September 2008; Accepted 23 January 2009

Recommended by Borut Zupancic

\begin{abstract}
A new mixed method for reducing the order of the large-scale linear dynamic multi-input-multi-output (MIMO) systems has been presented. In this method, the common denominator polynomial of the reduced-order transfer function matrix is synthesized by using modified pole clustering while the coefficients of the numerator elements are computed by minimizing the integral square error between the time responses of the original and reduced system element using Genetic Algorithm. The modified pole clustering generates more dominant cluster centres than cluster centres obtained by pole clustering technique already available in literature. The proposed algorithm is computer-oriented and comparable in quality. This method guarantees stability of the reduced model if the original high-order system is stable. The algorithm of the proposed method is illustrated with the help of an example and the results are compared with the other well-known reduction techniques.
\end{abstract}

Copyright (C) 2009 C. B. Vishwakarma and R. Prasad. This is an open access article distributed under the Creative Commons Attribution License, which permits unrestricted use, distribution, and reproduction in any medium, provided the original work is properly cited.

\section{Introduction}

Every physical system can be translated into mathematical model. The mathematical procedure of system modeling often leads to comprehensive description of a process in the form of high-order differential equations which are difficult to use either for analysis or controller synthesis. It is, therefore, useful, and sometimes necessary, to find the possibility of finding some equations of the same type but of lower order that may be considered to adequately reflect the dominant characteristics of the system under consideration. Some of the reasons for using reduced-order models of highorder linear systems could be as follows:

(i) to have a better understanding of the system,

(ii) to reduce computational complexity,

(iii) to reduce hardware complexity,

(iv) to make feasible controller design.

Several reduction methods are available in literature for reducing the order of large-scale linear MIMO systems in frequency domain [1-4]. Further, some mixed methods have been suggested by combining the algorithm of two different reduction methods [5-7]. In spite of having several reduction methods, none always gives the satisfactory results for all the systems.

The optimization-based reduced-order modeling has already been suggested in the work [8-10] in which the numerator coefficients are computed by minimizing the integral square error (ISE) between the step responses of the original and the reduced system while the denominator polynomial is obtained by using existing order-reduction technique.

The Genetic Algorithm (GA) is a popular optimization technique in the science and engineering nowadays. This is based on the process of Darwin 's Theory of Evolution. By starting with a set of potential solutions and changing them during several iterations, the GA hopes to converge on the most "fit" solution. The process begins with a set of potential solutions or chromosomes (usually in the form of bit strings) that are randomly generated or selected. The entire set of these chromosomes comprises a population. The chromosomes evolve during several iterations or generations. New generations (offspring) are generated using 
the crossover and mutation technique. Crossover involves splitting two chromosomes and then combining one half of each chromosome with the other pair. Mutation involves flipping a single bit of a chromosome. The chromosomes are then evaluated using a certain fitness criteria and the best ones are kept while the others are discarded. This process repeats until one chromosome has the best fitness and thus is taken as the best solution of the problem [11].

The authors suggested a modified pole clustering based on the concept of the clustering technique $[12,13]$ in which clusters of the poles of the original high-order system are formed and then each cluster is replaced by its cluster centre by using inverse distance measure criterion. The modified pole clustering technique generates more dominant cluster centre than the cluster centre obtained by authors $[12,13]$.

In the present study, the authors proposed a new mixed method for reducing the order of the linear MIMO systems in which the common denominator polynomial of the reduced system is obtained by using modified pole clustering while the coefficients of the numerator element of the reduced model are computed by using GA.

\section{Reduction Method}

Let the $n$ th-order transfer function matrix of the high-order original system having " $p$ " inputs and " $q$ " outputs be

$$
[G(s)]=\frac{1}{D_{n}(s)}\left[\begin{array}{cccc}
a_{11}(s) & a_{12}(s) & \cdots & a_{1 p}(s) \\
a_{21}(s) & a_{22}(s) & \cdots & a_{2 p}(s) \\
\vdots & \vdots & \vdots & \vdots \\
a_{q 1}(s) & a_{q 2}(s) & \cdots & a_{q p}(s)
\end{array}\right]
$$

or let $[G(s)]=\left[g_{i j}(s)\right], i=1,2, \ldots, q ; j=1,2, \ldots, p$ be a $q \times p$ transfer matrix.

The general form of $g_{i j}(s)$ can be taken as

$$
g_{i j}(s)=\frac{a_{i j}(s)}{D_{n}(s)}=\frac{a_{0}+a_{1} s+\cdots+a_{n-1} s^{n-1}}{b_{0}+b_{1} s+\cdots+b_{n} s^{n}},
$$

where $i=1,2, \ldots, q ; j=1,2, \ldots, p$.

Let the reduced transfer function matrix of the $k$ th-order having " $p$ " inputs and " $q$ " outputs to be synthesized as

$$
\left[R_{k}(s)\right]=\frac{1}{D_{k}(s)}\left[\begin{array}{cccc}
c_{11}(s) & c_{12}(s) & \cdots & c_{1 p}(s) \\
c_{21}(s) & c_{22}(s) & \cdots & c_{2 p}(s) \\
\vdots & \vdots & \vdots & \vdots \\
c_{q 1}(s) & c_{q 2}(s) & \cdots & c_{q p}(s)
\end{array}\right]
$$

or let $\left[R_{k}(s)\right]=\left[r_{i j}(s)\right], i=1,2, \ldots, q ; j=1,2, \ldots, p$ be a $q \times p$ transfer matrix.

The general form of $r_{i j}(s)$ can be taken as

$$
r_{i j}(s)=\frac{c_{i j}(s)}{D_{k}(s)}=\frac{c_{0}+c_{1} s+\cdots+c_{k-1} s^{k-1}}{d_{0}+d_{1} s+\cdots+d_{k} s^{k}},
$$

where $i=1,2, \ldots, q ; j=1,2, \ldots, p$.

The objective of this paper is to realize a reduced transfer matrix of the $k$ th-order in the form of (3) from the original high-order transfer matrix (1) such that it retains the important features of the original high-order system $[G(s)]$.

Further, the reduction procedure consists of the following two steps.

Step 1. Determination of the common denominator polynomial $D_{k}(s)$ for the reduced transfer matrix using modified pole clustering technique.

The brief algorithm for realizing the denominator polynomial by using the modified pole clustering is as follows.

Let there be $r$ real poles in the $i$ th cluster: $p_{1}, p_{2}, \ldots, p_{r}$, where $\left|p_{1}\right|<\left|p_{2}\right| \cdots<\left|p_{r}\right|$, and then modified cluster centre $p_{e i}$ can be obtained by using the algorithm of the modified pole clustering suggested in this paper.

Let $m$ pairs of complex conjugate poles in a $j$ th cluster be

$$
\left[\left(\alpha_{1} \pm j \beta_{1}\right),\left(\alpha_{2} \pm j \beta_{2}\right), \ldots,\left(\alpha_{m} \pm j \beta_{m}\right)\right]
$$

where $\left|\alpha_{1}\right|<\left|\alpha_{2}\right| \cdots<\left|\alpha_{m}\right|$.

Now using the same algorithm separately for real and imaginary parts of the complex conjugate poles, the modified cluster centre is obtained as

$$
\Phi_{e j}=A_{e j} \pm j B_{e j}
$$

where $\stackrel{*}{\Phi}_{e j}=A_{e j}+j B_{e j}$ and $\stackrel{\circ}{\Phi}_{e j}=A_{e j}-j B_{e j}$.

An interactive computer programme has been developed that automatically finds the modified cluster centre and is given as follows:

(1) let $r$ number of the real poles in a cluster be $\left|p_{1}\right|<$ $\left|p_{2}\right| \cdots<\left|p_{r}\right|$

(2) set $j=1$,

(3) find pole cluster centre by $c_{j}=\left[\sum_{i=1}^{r}\left(-1 /\left|p_{i}\right|\right) \div r\right]^{-1}$,

(4) set $j=j+1$,

(5) now find a modified cluster centre from $c_{j}=[(-1 /$ $\left.\left.\left|p_{1}\right|+-1 /\left|c_{j-1}\right|\right) \div 2\right]^{-1}$,

(6) is $j=r$ ?, if No, then go to step (4) otherwise go to step (7),

(7) take a modified cluster centre of the $k$ th-cluster as $p_{e k}=c_{j}$.

For synthesizing the common denominator polynomial, one of the following cases may occur.

Case 1. If all the modified cluster centres are real, then denominator polynomial of the $k$ th-order reduced model can be obtained as

$$
D_{k}(s)=\left(s-p_{e 1}\right)\left(s-p_{e 2}\right) \cdots\left(s-p_{e k}\right),
$$

where $p_{e 1}, p_{e 2}, \ldots, p_{e k}$ are the first-, second-,.., $k$ thmodified cluster centre, respectively. 
Case 2. If all the modified cluster centres are complex conjugate, then the $k$ th-order denominator polynomial is taken as

$$
D_{k}(s)=\left(s-\stackrel{*}{\Phi}_{e 1}\right)\left(s-\stackrel{\circ}{\Phi}_{e 1}\right) \cdots\left(s-\stackrel{*}{\Phi}_{e k / 2}\right)\left(s-\stackrel{\circ}{\Phi}_{e k / 2}\right) .
$$

Case 3. If some cluster centres are real and some are complex conjugate, then this case holds.

Let $(k-2)$ cluster centres are real and one pair of cluster centre is complex conjugate, then the $k$ th-order denominator can be obtained as

$D_{k}(s)=\left(s-p_{e 1}\right)\left(s-p_{e 2}\right) \cdots\left(s-p_{e(k-2)}\right)\left(s-\stackrel{*}{\Phi}_{e 1}\right)\left(s-\stackrel{\circ}{\Phi}_{e 1}\right)$.

Step 2. Determination of the numerator $c_{i j}(s)$ of the reduced transfer matrix $r_{i j}(s)$ using GA.

In this paper, GA has been applied to minimize the objective function " $F_{i j}$," which is an integral square error ISE [9] between the unit step responses of the elements of the original and the reduced system and is given by

$F_{i j}=\int_{0}^{\infty}\left[g_{i j}(t)-r_{i j}(t)\right]^{2} d t, \quad i=1,2, \ldots, q ; j=1,2, \ldots, p$,

where $g_{i j}(t)$ and $r_{i j}(t)$ are the unit step responses of the elements of original high-order system $[G(s)]$ and the reduced-order model $\left[R_{k}(s)\right]$, respectively. The coefficients $c_{j} ; 1 \leq j \leq k-1$ of the numerator element $c_{i j}(s)$ are determined with suitable GA parameters.

To ensure the zero steady-state error, the coefficient $c_{0}$ is obtained as

$$
c_{0}=\frac{a_{0}}{b_{0}} \cdot d_{0} .
$$

The computational flowchart of the GA optimization is shown in Figure 1.

\section{Numerical Example}

To demonstrate the accuracy and usefulness of the proposed method, one numerical example is taken from the literature and the second-order reduced model is obtained using the suggested algorithm.

Consider a sixth-order system [2] having two-input and two-output described by the transfer matrix:

$$
\begin{aligned}
{[G(s)] } & =\left[\begin{array}{cc}
\frac{2(s+5)}{(s+1)(s+10)} & \frac{(s+4)}{(s+2)(s+5)} \\
\frac{(s+10)}{(s+1)(s+20)} & \frac{(s+6)}{(s+2)(s+3)}
\end{array}\right], \\
& =\frac{1}{D_{6}(s)}\left[\begin{array}{ll}
a_{11}(s) & a_{12}(s) \\
a_{21}(s) & a_{22}(s)
\end{array}\right]
\end{aligned}
$$

where $D(s)$ is the common denominator polynomial.

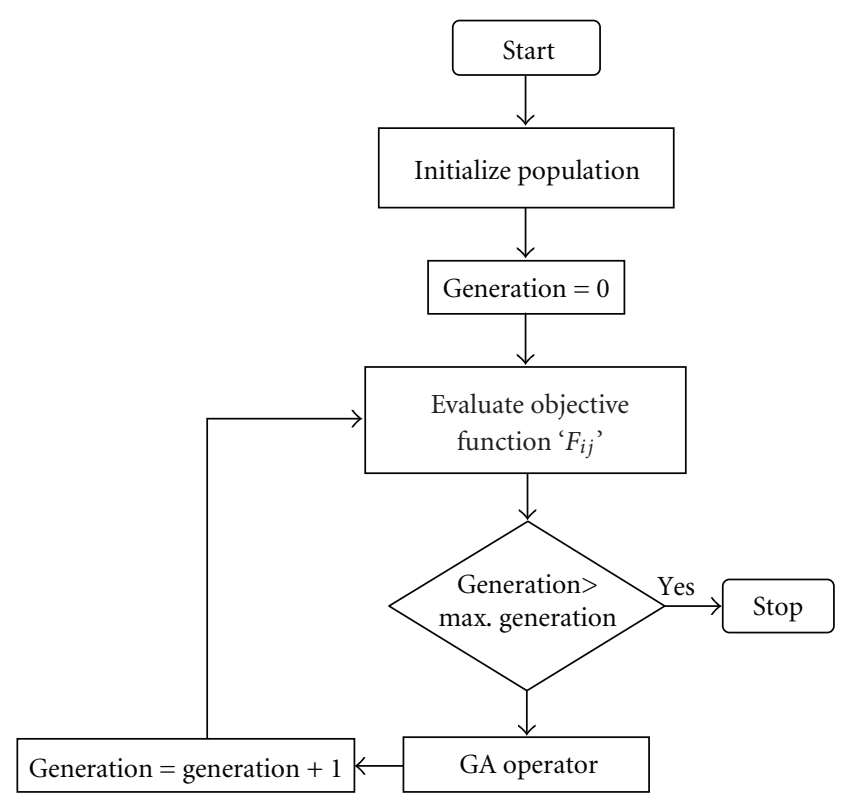

FIGURE 1: Flowchart of genetic algorithm.

The poles are $-1,-2,-3,-5,-10,-20$. Let the first and second clusters contain the poles $-1,-2$ and $-3,-5,-10$, -20 , respectively for getting second-order reduced model.

Now using Step 1, the modified cluster centres are obtained as

$$
p_{e 1}=-1.1428, \quad p_{e 2}=-3.1946 .
$$

Using Case 1, the denominator polynomial for the secondorder reduced model is obtained as

$$
D_{2}(s)=(s+1.1428)(s+3.1946)=3.65079+4.3374 s+s^{2} .
$$

Step 2 of the proposed algorithm is successively applied to each element of the high-order original MIMO system and the reduced models $r_{i j}(s)$ of the $\left[R_{2}(s)\right]$ and then $c_{i j}(s): 1 \leq$ $i \leq 2 ; 1 \leq j \leq 2$ are obtained as

$$
\begin{aligned}
& c_{11}(s)=3.65079+1.18156 s, \\
& c_{12}(s)=1.46031+1.04664 s, \\
& c_{21}(s)=1.82539+0.49819 s, \\
& c_{22}(s)=3.65079+1.6911 s .
\end{aligned}
$$

Therefore, finally the second-order reduced model is obtained as

$$
\begin{aligned}
{\left[R_{2}(s)\right]=} & \frac{1}{3.65079+4.3374 s+s^{2}} \\
& \times\left[\begin{array}{ll}
3.65079+1.18156 s & 1.46031+1.04664 s \\
1.82539+0.49819 s & 3.65079+1.6911 s
\end{array}\right] .
\end{aligned}
$$

The step responses of the reduced-order models $r_{i j}(s)$ are compared with the original system $g_{i j}(s)$ and are shown in Figure 2 and the proposed method is compared with the other well-known order-reduction techniques available in literature and are shown in the Table 1 by comparing 
TABLE 1: Comparison of reduction methods.

\begin{tabular}{lcccc}
\hline & & ISE for $r_{i j}(i=1,2 ; j=1,2)$ & & $r_{21}$ \\
\hline Reduction method & $r_{11}$ & $r_{12}$ & 0.000299 & 0.004681 \\
\hline Proposed method & 0.001515 & $7.845 \times 10^{-5}$ & 0.002538 & 0.015741 \\
Parmar et al. [9] & 0.014498 & 0.008744 & 0.040291 & 0.067902 \\
Prasad and Pal [14] & 0.136484 & 0.002446 & 0.007328 & 1.066123 \\
Safonov and Chiang [15] & 0.590617 & 0.037129 & 0.261963 & 0.021683 \\
Prasad et al. [1] & 0.030689 & 0.000256 & & \\
\hline
\end{tabular}

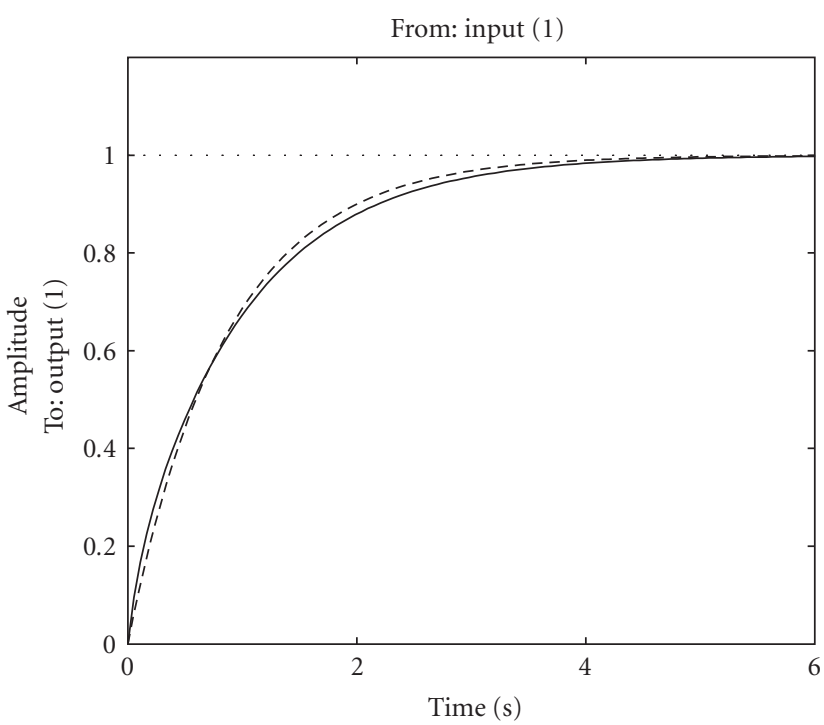

- Original system

- - - Reduced model

(a)

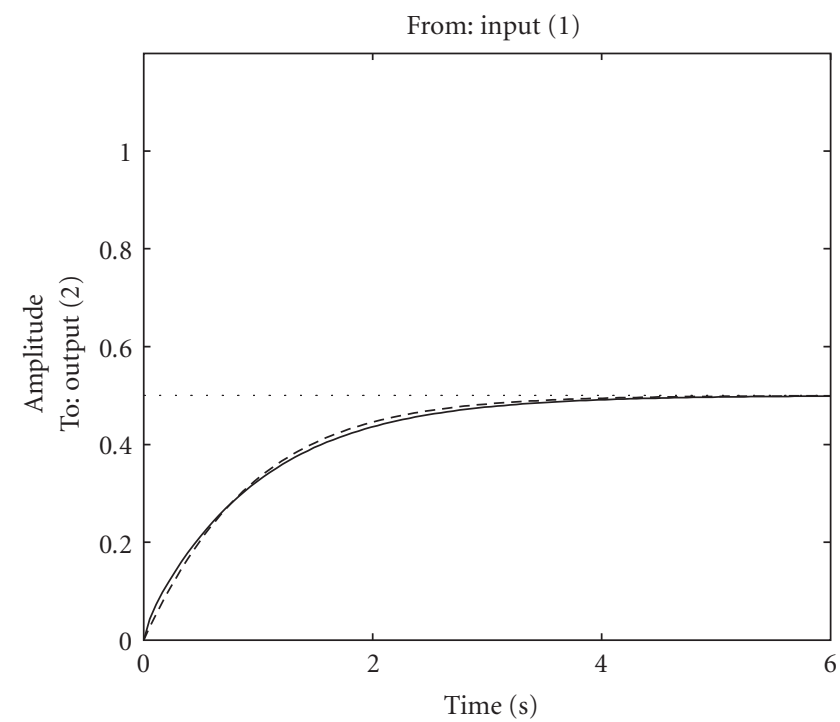

- Original system

- - - Reduced model

(c)

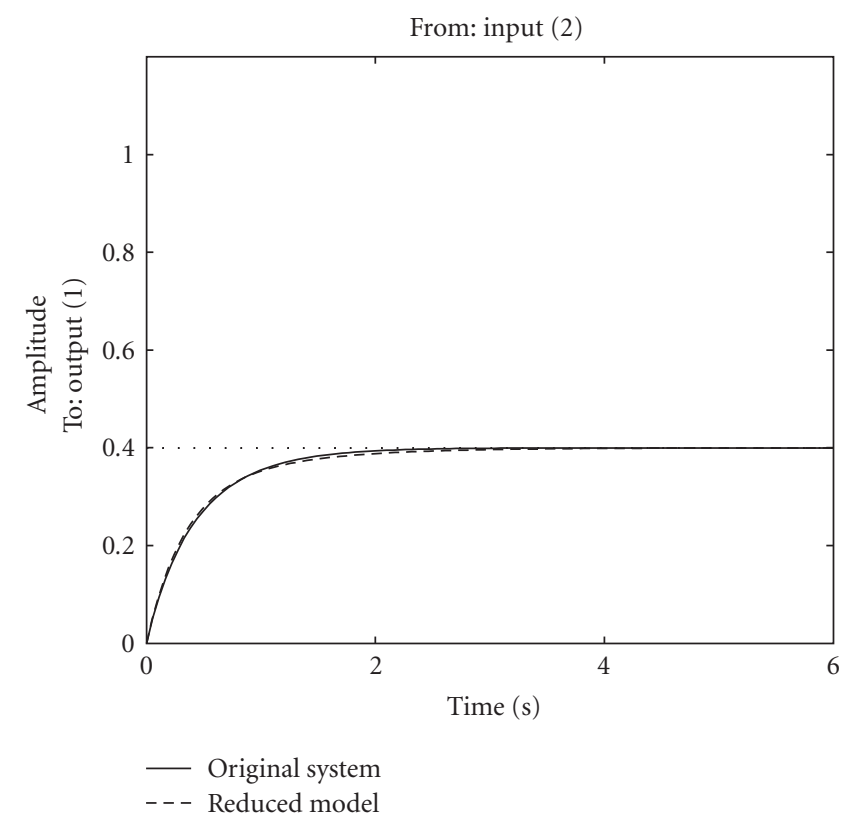

(b)

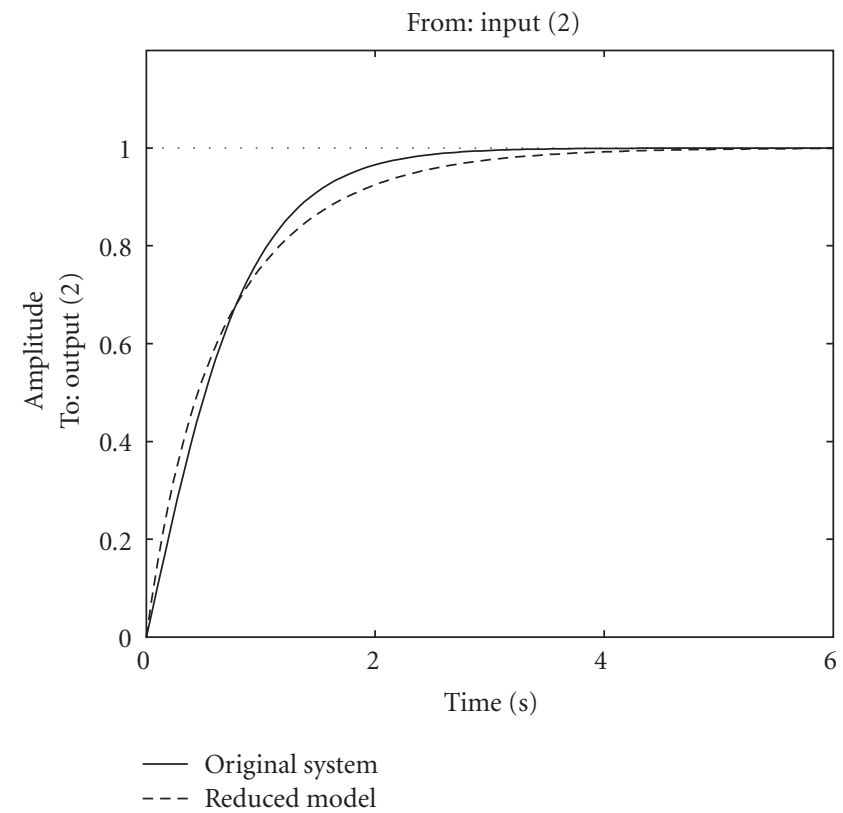

(d)

Figure 2: Comparison of the step responses. 
the integral square error ISE in between the step responses of the original $g_{i j}(s)$ and reduced $r_{i j}(s)$-order systems. The ISE is given as

$$
\mathrm{ISE}=\int_{0}^{\infty}\left[g_{i j}(t)-r_{i j}(t)\right]^{2} d t
$$

where, $i=1,2, j=1,2$ and $g_{i j}(t), r_{i j}(t)$ are the unit step responses of the original and reduced systems respectively for $i$ th-output with $j$ th-input.

\section{Conclusions}

A new mixed method which combines the advantages of the modified pole clustering and the integral square error minimization by using GA has been presented to derive stable reduced-order models of the linear time-invariant MIMO systems.

In this method, the common denominator polynomial of the reduced transfer function matrix is synthesized using the modified pole clustering, and the coefficients of the numerator of the reduced model are computed by minimizing the integral square error between the step responses of the original and reduced system using GA. The method is simple, rugged, and computer-oriented. The proposed algorithm has been applied on the system having two inputs and two outputs to get second-order reduced model. The comparison of the step responses of the original and reduced system is shown in Figure 2 and ISE is calculated between the original and reduced system and compared in the tabular form as given in Table 1, from which it is clear that the proposed method is comparable in quality with the other well-known order-reduction techniques for MIMO systems. The method also preserves the model stability and avoids any steady-state error between the time responses of the original and reduced systems. The proposed method may be extended to unstable systems and is reported elsewhere.

\section{References}

[1] R. Prasad, J. Pal, and A. K. Pant, "Multivariable system approximation using polynomial derivatives," Journal of the Institution of Engineers, vol. 76, pp. 186-188, 1995.

[2] Y. Bistritz and U. Shaked, "Minimal Pade model reduction for multivariable systems," Journal of Dynamic Systems, Measurement and Control, vol. 106, no. 4, pp. 293-299, 1984.

[3] C. F. Chen, "Model reduction of multivariable control systems by means of matrix continued fractions," International Journal of Control, vol. 20, no. 2, pp. 225-238, 1974.

[4] M. R. Calfe and M. Healey, "Continued fraction model reduction technique for multivariable systems," Proceedings of the Institution of Electrical Engineers, vol. 121, no. 5, pp. 393395, 1974.

[5] R. Prasad, "Pade type model order reduction for multivariable systems using routh approximation," Computers \& Electrical Engineering, vol. 26, no. 6, pp. 445-459, 2000.

[6] R. Prasad, A. K. Mittal, and S. P. Sharma, "A mixed method for the reduction of multi-variable systems," Journal of the Institution of Engineers, vol. 85, pp. 177-181, 2005.
[7] L. Shieh and Y. Wei, "A mixed method for multivariable system reduction," IEEE Transactions on Automatic Control, vol. 20, no. 3, pp. 429-432, 1975.

[8] S. Mukherjee and R. N. Mishra, "Reduced order modelling of linear multivariable systems using an error minimization technique," Journal of the Franklin Institute, vol. 325, no. 2, pp. 235-245, 1988.

[9] G. Parmar, R. Prasad, and S. Mukherjee, "Order reduction of linear dynamic systems using stability equation method and GA," International Journal of Computer, Information, and Systems Science, and Engineering, vol. 1, no. 1, pp. 26-32, 2007.

[10] S. S. Lamba, R. Gorez, and B. Bandyopadhyay, "New reduction technique by step error minimization for multivariable systems," International Journal of Systems Science, vol. 19, no. 6, pp. 999-1009, 1988.

[11] Y. Zheng and S. Kiyooka, "Genetic Algorithms Applications: Assignment \#2 for Dr. Z. Dong," November 1999, http:// www.me.uvic.ca/ zdong/courses/mech620/GA_App.PDF.

[12] J. Pal, A. K. Sinha, and N. K. Sinha, "Reduced order modelling using pole-clustering and time-moment matching," Journal of the Institution of Engineers, vol. 76, pp. 1-6, 1995.

[13] A. K. Sinha and J. Pal, "Simulation based reduced order modelling using a clustering technique," Computers \& Electrical Engineering, vol. 16, no. 3, pp. 159-169, 1990.

[14] R. Prasad and J. Pal, "Use of continued fraction expansion for stable reduction of linear multivariable systems," Journal of the Institution of Engineers, vol. 72, pp. 43-47, 1991.

[15] M. G. Safonov and R. Y. Chiang, "Model reduction for robust control: a schur relative error method," International Journal of Adaptive Control and Signal Processing, vol. 2, no. 4, pp. 259272, 1988. 

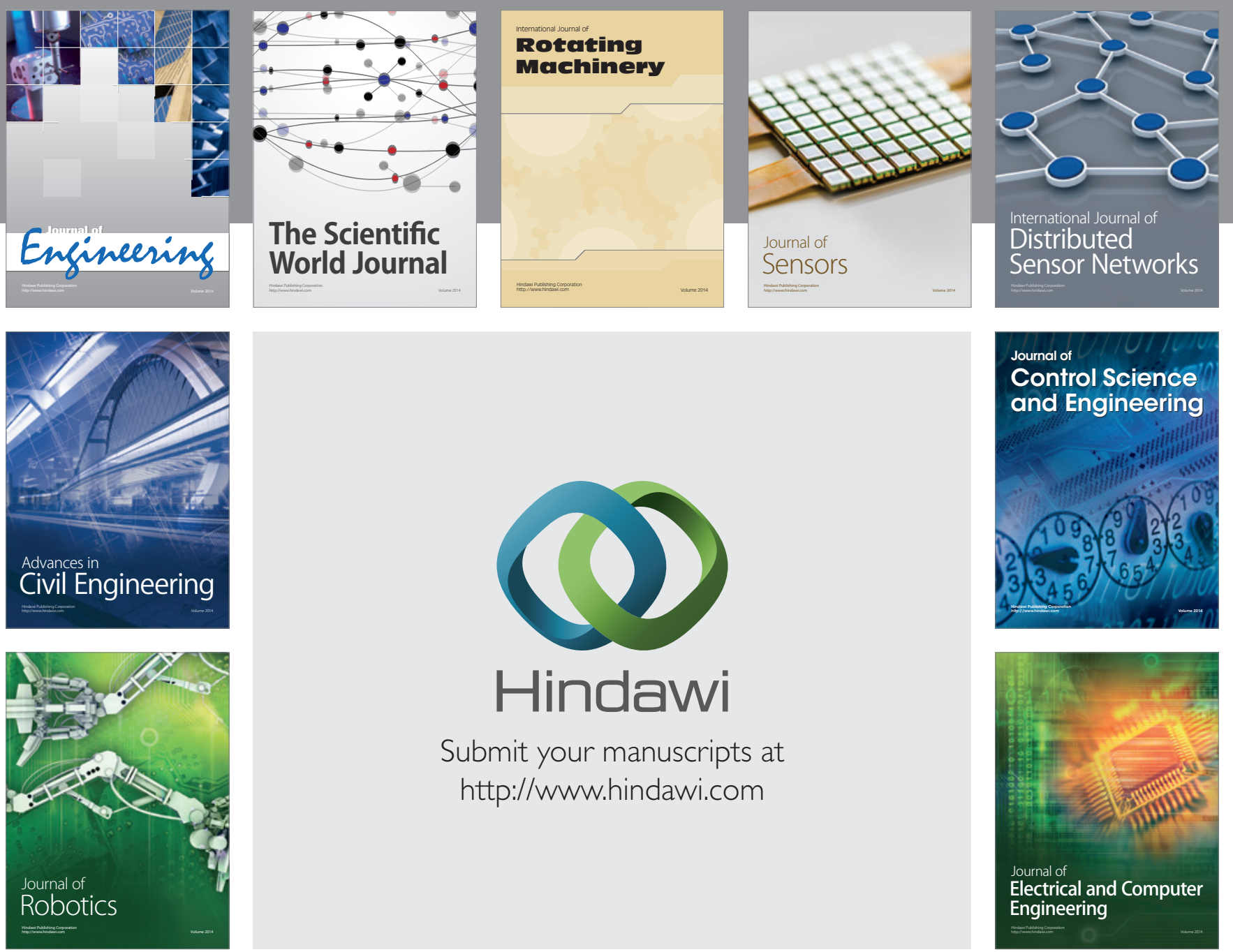

Submit your manuscripts at

http://www.hindawi.com
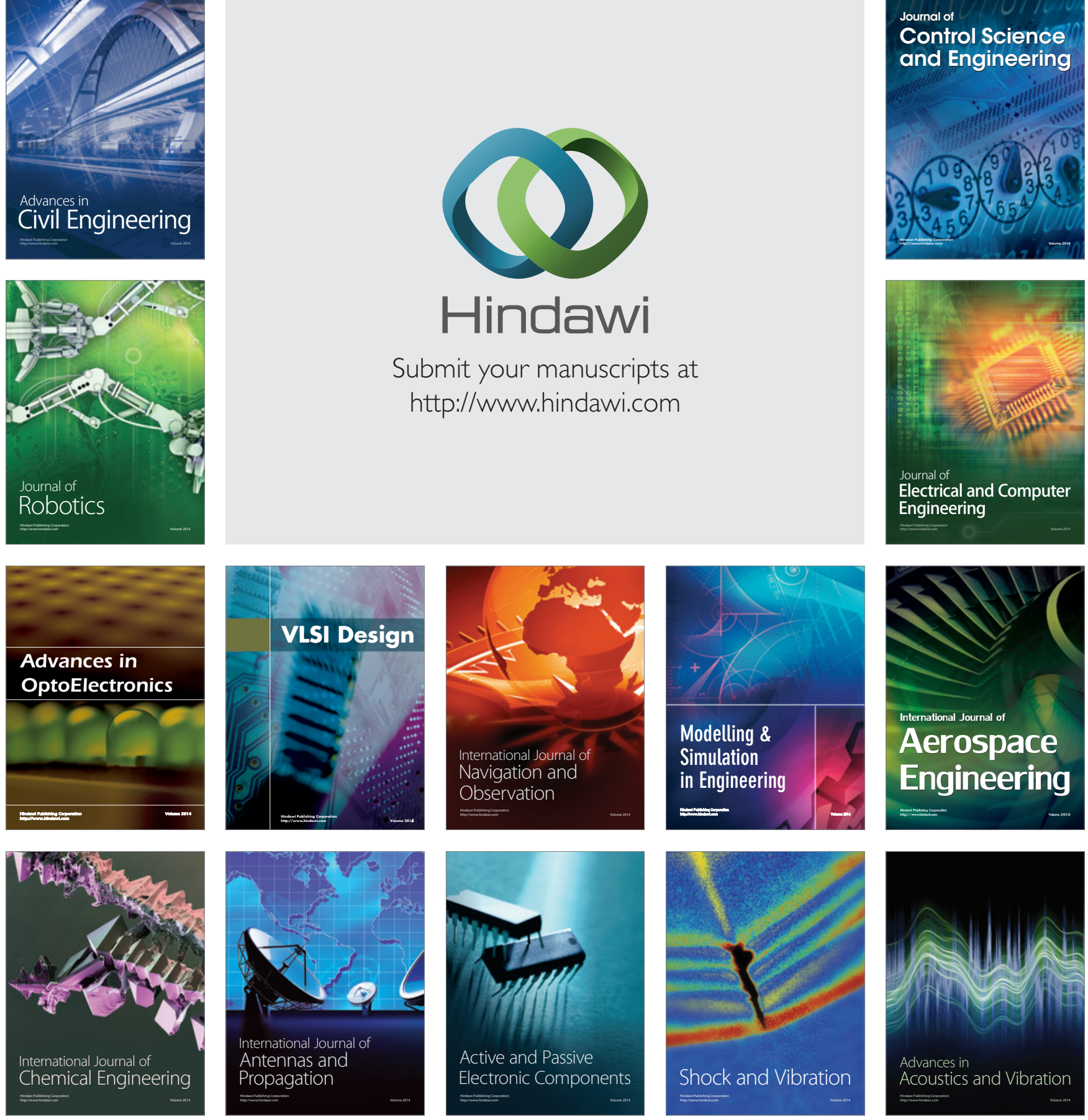